\title{
Novel Application of the Dye, DiS-C3-(3) as a Biosensor of the Renal Membrane Potential Difference
}

\author{
Othman A. Alghamdi and Nicola King
}

\begin{abstract}
The generation and maintenance of the membrane potential difference is of fundamental importance to the livelihood of every cell. The aim of this study was to show for the first time that the fluorescent indicator,

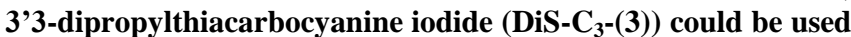
as a reporter of the membrane potential difference in various membrane vesicle populations isolated from the rat kidney. When thesemembrane vesicles were added to a solution that imposed different transmembrane potassium gradients the measured change in fluorescence $(\Delta F)$ was dependent upon the predicted membrane potential difference. The more positive the membrane potential difference, the smaller the $\Delta \mathrm{F}$ measured. To illustrate the potential applicability of this dye for measuring membrane transport phenomena it was shown that DiS- $_{3}$-(3)fluorescence was sensitive to the more subtle changes in membrane potential evoked by the electrogenic transport activities associated with the $\mathrm{H}^{+}$-coupled uptake of L-glycyl-L-glutamine into brush border membrane vesicles isolated from the rat kidney. These findings demonstrate the suitability of $\mathrm{DiS}_{-} \mathrm{C}_{3^{-}}(3)$ as a biosensor of the membrane potential difference with scope for a broad range of applications.
\end{abstract}

Index Terms-3'3-dipropylthiacarbocyanine iodide, fluorescent spectroscopy, membrane potential difference, membrane transport.

\section{INTRODUCTION}

The generation and maintenance of a membrane potential difference is fundamentally important to many physiological processes. For example the inherent instability of the membrane potential difference of cells located in the sinoatrial node in the heart enables these cells to determine the pacemaker potential that sets the heart's rhythm and rate [1]. Equally significant, is the energy produced as electrons are passed from one carrier to the next in the mitochondria, which facilitates the transfer of protons across the inner mitochondrial membrane generating an electrochemical gradient that when the protons are returned powers ATP synthesis [2]. Amongst other things, this ATP provides energy for the $\mathrm{Na}^{+}-\mathrm{K}^{+}$-ATPase, found in all cells, which actively pumps potassium ions into the cell in exchange for the extrusion of sodium ions [3]. This creates an electrochemical gradient favoring potassium exit from the cell leading to the generation of an inside negative membrane

Manuscript received June 25, 2012; revised July 30, 2012. This work was supported by the University of New England. O. A. Alghamdi was supported by the Saudi Ministry of Higher Education.

Both O. A. Alghamdi and N. King are with the School of Science and Technology, McClymont Building, University of New England, Armidale, NSW 2351. Australia (e-mail: oalghamd@une.edu.au; nking20@une.edu.au, phone: +61-2-6673-2579, Fax: +61-2-6773-3267) potential difference and an inward sodium gradient since sodium is the most abundant extracellular cation. Combined together the kinetic energy liberated from this electrochemical gradient provides the driving force that enables the efficient absorption of many vital nutrients and which can be harnessed for drug delivery [3]. Therefore the development of efficient methods for measuring and possibly manipulating the membrane potential difference could have important implications for understanding the complexities of nutrient handling and pharmokinetics.

The reabsorption of amino acids in the kidney is essential to body protein balance. In the kidney proximal tubule the proton-coupled absorption of small peptides containing 2-3 amino acids by PEPT1and PEPT2is a dynamic process in which translocation is forced by a transmembrane electrochemical proton gradient [4]-[5]. The apically located $\mathrm{Na}^{+}-\mathrm{H}^{+}$exchanger NHE3 helps to acidify the lumen to approximately $\mathrm{pH}$ 6.7-6.8 [6] by progressively secreting protons $\left(\mathrm{H}^{+}\right)$across the brush border membrane [7]-[8]. This creates an inward $\mathrm{H}^{+}$electrochemical gradient, which the PEPT cotransportersutilise to force the uphill accumulation of any of the 4000 different naturally occurring dipeptides and 8000 possible tripeptide combinations into the proximal tubule cells [7]. Understanding the mechanism of these transporters also has important pharmacological implications, as, based on structural mimicry, the PEPT cotransporterswill also accept any molecule that has a basic peptide-like structure with classical examples including numerous $\beta$-lactam antibiotics of the cephalosporin (e.g. cefadroxil) and penicillin (e.g. ampicillin) classes; selected angiotensin converting enzyme inhibitors (e.g. captopril); and a few anti-tumor drugs (e.g. $\delta$-amino levulinic acid and bestatin) [7].

In this paper we show for the first time that the fluorescent indicator, 3'3'-dipropylthiacarbocyanine iodide (Dis-C $\left.\mathrm{C}_{3}-(3)\right)$ can be used to sense the membrane potential difference of the renal proximal tubule brush border membrane. In a further novel illustration of the uses of this dye we then proceed to demonstrate how it can be applied to the measurement of the uptake of the dipeptide ${ }_{\mathrm{L}}$-glycyl${ }_{{ }_{\mathrm{L}}}$-glutamine (gly-gln) in brush border membrane vesicles isolated from the rat kidney.

\section{MATERIALS AND METHODS}

\section{A. Chemicals and Tissue}

All chemicals and reagents used in this study were obtained from SIGMA, Ajax Finechem Pty Ltd, Chem-Supply or Applichem and were of the best analytical quality available. Kidneys were obtained from male Wistar rats of either 6-8 weeks (young) or 8-9 months (middle-aged) old. The rats were sacrificed by stunning and cervical 
dislocation. The kidneys were snap frozen in liquid nitrogen and placed at $-80^{\circ} \mathrm{C}$ until required for the preparation of membrane vesicles. This study was approved by the local Animal Ethics Committee and conforms to the Guide for the care and use of laboratory Animals published by the US National Institute of Health (NIH Publication No. 85-23, revised 1996).

\section{B. Isolation of Membrane Vesicles}

The isolation procedure used to prepare brush border membrane vesicles from the superficial cortex (BBMV) and outer medulla (OMMV) was a dual-stage magnesium precipitation technique as described previously [9]. The quality of the purified vesicles was assessed using marker enzyme assays (leucineaminopeptidase and alkaline phosphatase, results not shown) and the protein concentration was measured using Bradford's method as described previously [9]. Prepared vesicles were stored at $-80^{\circ} \mathrm{C}$ until use.

\section{Optimisation of DiS-C3-(3) for Fluorescentmeasurements}

An initial stock solution containing $114.7 \mu \mathrm{M} \mathrm{DiS-C}{ }_{3}-(3)$ was prepared in dimethylformide and stored at $4^{\circ} \mathrm{C}$ until use. The concentration of this stock was verified using the Beer Lambert law combined with the absorption of the stock at $556 \mathrm{~nm}$ and the extinction coefficient ofthe dye.

All experiments were carried out at room temperature using a Fluorescence spectrophotometer (ISS, $\mathrm{K}_{2}$ Multifrequency Cross-correlation phase and modulation fluorimeter) pre-set with: $8 \mathrm{~mm}$ slit width, excitation and emission wavelengths of 557 and $575 \mathrm{~nm}$ respectively; and the cuvette used was $0.2 \mathrm{~mm}$. Fluorescence intensity (in arbitrary units) was recorded at 10 second intervals for 500 seconds and displayed on a digital screen as a plot of fluorescence versus time. In order to ascertain the optimal excitation and emission wavelength for $\mathrm{DiS}_{-} \mathrm{C}_{3}-(3)$ measurements were made where changes in fluorescence were recorded when the excitation wavelength was varied between 570-700nm, whilst the emission wavelength was held at 556nm; and when the excitation wavelength was held at $575 \mathrm{~nm}$ and the emission wavelength was varied between $400-565 \mathrm{~nm}$. The results as shown in Fig. 1 clearly indicated that the optimal excitation and emission wavelengths to use with Dis- $\mathrm{C}_{3}-(3)$ were $556 \mathrm{~nm}$ and $575 \mathrm{~nm}$ respectively.

\section{Measurement of the Transmembrane Potential \\ Difference with Different Potassium Gradients and in the Presence of Gly-Gln}

Fluorescence intensity (in arbitrary units) was recorded onceevery 10 second overa 500 second period. The fluorescence measurement was displayed on a digital screen.

Experiments began by pipetting the appropriate volume of buffer and $8.6 \mu \mathrm{l}(3.3 \mu \mathrm{M})$ of $\mathrm{DiS}_{-} \mathrm{C}_{3}-(3)$ into a cuvette. The cuvette was placed into the fluorimeter and the fluorescence followed for 200 seconds to enable stabilisation of the signal. An aliquot of membrane vesicles was then added to the cuvette in order that the final volume was $300 \mu$ l containing $0.027 \mathrm{mg}$ of vesicle protein (maintaining a dye to protein ratio of $19.4 \mu \mathrm{g} / \mathrm{mg}$ protein) [10]-[11] and the fluorescence recorded for a further 300s. Graphs with the fluorescence intensity (in arbitrary units) shown over time (in seconds) were obtained by incorporation into Vinci software (BETA 1-7) in an IBM compatible computer.

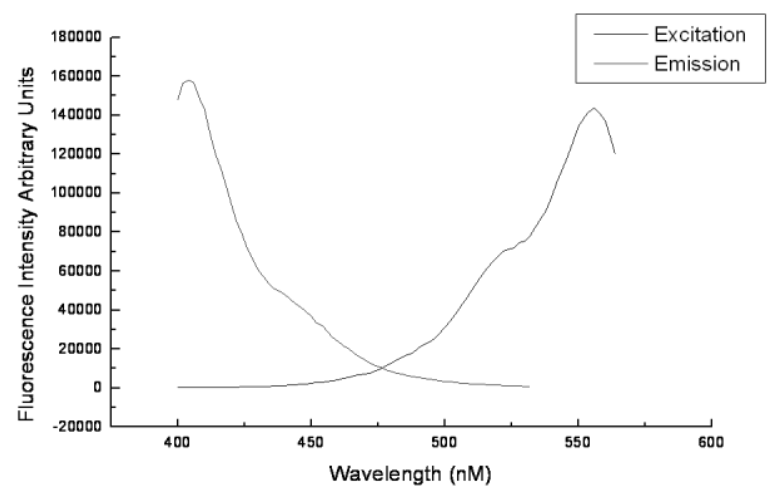

Fig. 1. Emission and excitation spectra of $\mathrm{DiS}_{-} \mathrm{C}_{3}-(3)$. Changes in fluorescence measured at different emission wavelengths, whilst the excitation wavelength was held at $556 \mathrm{~nm}$ (line on the left), and at different excitation wavelengths, whilst the emission wavelength was held at $575 \mathrm{~nm}$ (line on the right)

In order to assess the sensitivity of $\mathrm{DiS}-\mathrm{C}_{3}-(3)$ as a reporter of the intravesicular membrane potential difference, a series of different $\mathrm{K}^{+}$diffusion potentials were established across the vesicle membranes. To achieve this, the vesicles were removed from the freezer, thawed on ice, centrifuged at $16,000 \mathrm{rpm}$ for $20 \mathrm{~min}$ at $4^{\circ} \mathrm{C}$ and then pre-equilibrated with a solution containing (in mM): 298 mannitol, $1 \mathrm{KH}_{2} \mathrm{PO}_{4}$ and 10 HEPES ( $\mathrm{pH} 7.4$ with TRIS) using freeze-thaw fractionation [12]. Changes in fluorescence were measured as described above when these vesicles were added to a cuvette containing $\mathrm{DiS}_{-} \mathrm{C}_{3}-(3)$ and one of three extravesicular solutions. More specifically, these extravesicular solutions were (in mM): (1) 298 mannitol, $1 \mathrm{KH}_{2} \mathrm{PO}_{4}$ and $10 \mathrm{HEPES}$ (pH 7.4 with TRIS); (2) 280 mannitol, $10 \mathrm{KH}_{2} \mathrm{PO}_{4}$ and 10 HEPES (pH 7.4 with TRIS); (3) 100 mannitol $100 \mathrm{KH}_{2} \mathrm{PO}_{4}$ and, 10 HEPES (pH 7.4 with TRIS). The presumed membrane potential difference in each of these conditions was calculated using the Nernst equation based on the assumption that the vesicles were freely permeable to potassium ions but would exclude phosphate ions [13].

BBMV and OMMV were defrosted on ice, suspended in a small volume of a medium containing (in $\mathrm{mM}$ ): 300 mannitol and 10 HEPES (pH 7.4 with TRIS) and centrifuged at 16,000 $\mathrm{RPM}$ and $4^{\circ} \mathrm{C}$ for $20 \mathrm{~min}$. The supernatant was discarded and the pellet was pre-equilibrated using freeze-thaw fractionation in a medium containing (in $\mathrm{mM}$ ): 100 mannitol, $100 \mathrm{KH}_{2} \mathrm{PO}_{4}$, and 10 HEPES (pH 7.4 with TRIS). The transport medium (i.e. the medium placed first into the cuvette along with the dye) contained (in $\mathrm{mM}$ ): 90-100 mannitol, $100 \mathrm{KH}_{2} \mathrm{PO}_{4}, 0-10$ L-glycyl-L-glutamine (gly-gln) and 10 2-(N-Morpholino)ethanesulfonic acid (MES, pH 6.0 with TRIS).

\section{E. Data Analysis and Presentation}

Unless otherwise stated, all of the results shown are means \pm SE of $n=4$ experiments. Statistical analyses were performed using one way analysis of variance (ANOVA with a Tukey post-test). $P$ values $<0.05$ were considered to be statistically significant. Graphs of the time course of fluorescence vs. time obtained with the VINCI software were directly incorporated into Microsoft Office by using the print screen and paste functions in the relevant software. 


\section{RESULTS}

In order to test the sensitivity of DiS- $\mathrm{C}_{3}$-(3) for reporting changes in the membrane potential difference in membrane vesicles isolated from rat kidneys, a time course of the fluorescence was measured in the presence of different transmembrane potassium gradients. The different potassium gradients used were respectively intravesicular to extravesicular: 1:1, 1:10 and 1:100. Fig. 2-4 show results from representative experiments using these parameters andouter medulla membrane vesicles isolated from middle-aged rats. There was a similar pattern to each of these Fig. starting with an initial stable period of fluorescence (approximately lasting 200s) when the cuvette contained just extravesicular medium and dye, a sharp increase in fluorescence as soon as the vesicles were added, followed by a plateau with a largely unchanging fluorescence level that continued for the remainder of the experiment (another 300s). This general pattern of a stable period, succeeded by a sharp increase and then a final largely unchanging plateauwas repeated in all vesicles populations (results not shown).

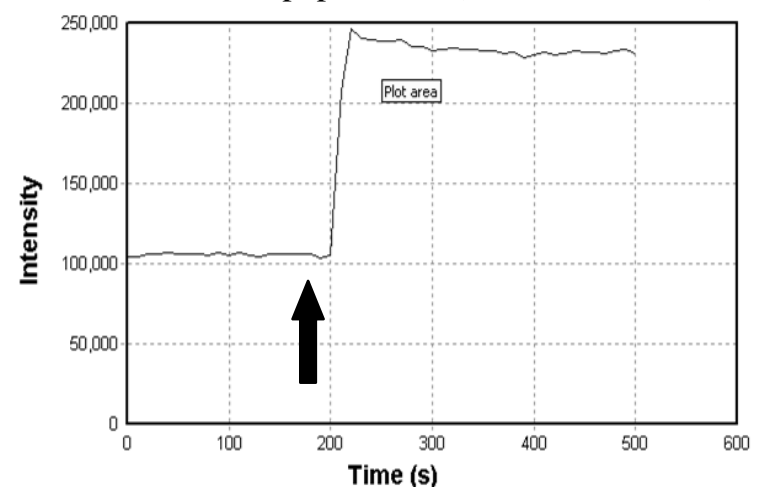

Fig. 2. Time course of the changes in fluorescence intensity (in arbitrary units) measured when OMMV isolated from middle-aged rats and pre-equilibrated with $1 \mathrm{mM} \mathrm{KH} \mathrm{KH}_{2} \mathrm{PO}_{4}$ were added to a cuvette containing DiS- $\mathrm{C}_{3}-(3)$ and $1 \mathrm{mM} \mathrm{KH}_{2} \mathrm{PO}_{4}$.

The black arrow indicates the time point at which the vesicles were added to the cuvette. Data shown are taken from a single experiment representative of 4 such experiments.

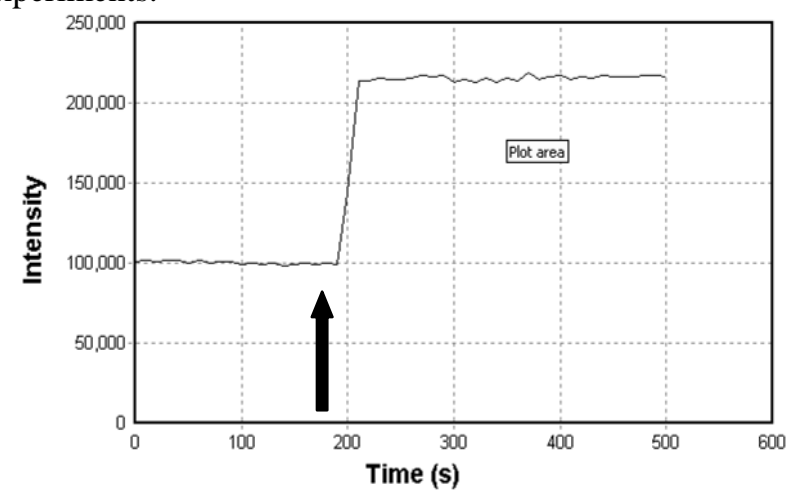

Fig. 3. Time course of the changes in fluorescence intensity measured when OMMV isolated from middle-aged rats and pre-equilibrated with $1 \mathrm{mM}$ $\mathrm{KH}_{2} \mathrm{PO}_{4}$ were added to a cuvette containing $10 \mathrm{mM} \mathrm{KH}_{2} \mathrm{PO}_{4}$ and DiS-C $\mathrm{C}_{3}$-(3).Other details as Fig. 2.

In order to comparethe changes in fluorescence when the OMMV were added to each of the different extravesicular potassium concentrations with changes in the vesicular membrane potential difference $\left(E_{m}\right)$, the $E_{m}$ in $\mathrm{mV}$ predicted for each transmembrane potassium gradient was calculated using the Nernst equation (1), as follows:

$$
E m=\frac{R T}{F} \operatorname{In} \frac{\left[K^{+}\right]_{0}}{\left[K^{+}\right]_{i}}
$$

where $R$ is the universal gas constant; $T$ is the absolute temperature; $\left[K^{+}\right]_{I}$ and $\left[K^{+}\right]_{0}$ are the intravesicular and extravesicular concentrations of potassium respectively; and $\mathrm{F}$ is the Faraday constant. This is based on the assumption that each vesicle population was freely permeable to potassium, but would exclude phosphate ions [13]. After rearranging this equation and changing the natural $\log$ to $\log$ base 10 , for an experiment at $23^{\circ} \mathrm{C}$, this may be rephrased (2) as:

$$
E m=58 \log \frac{\left[K^{+}\right]_{0}}{\left[K^{+}\right]_{i}}
$$

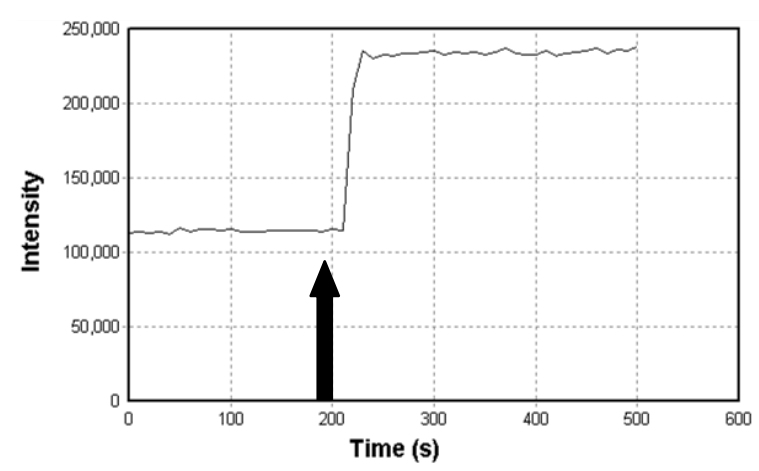

Fig. 4. Time course of the changes in fluorescence intensity measured when $\mathrm{OMMV}$ isolated from middle-aged rats and pre-equilibrated with $1 \mathrm{mM}$ $\mathrm{KH}_{2} \mathrm{PO}_{4}$ were added to a cuvette containing $100 \mathrm{mM} \mathrm{KH} \mathrm{KH}_{2} \mathrm{PO}_{4}$ and DiS-C ${ }_{3}$-(3).All other details as Fig. 2.

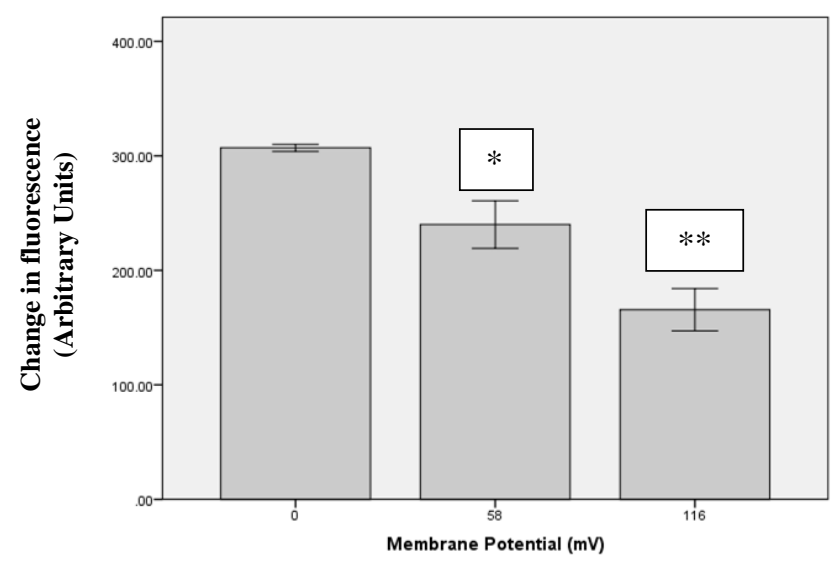

Fig. 5. Changes in fluorescence intensity measured when OMMV isolated from young kidneys and pre-equilibrated with $1 \mathrm{mM} \mathrm{KH}_{2} \mathrm{PO}_{4}$ were added to a cuvette containing DiS- $\mathrm{C}_{3}-(3)$ and 1,10 or $100 \mathrm{mM} \mathrm{KH}_{2} \mathrm{PO}_{4}$

$\mathrm{X}$-axis values were calculated from the Nernst equation (1). Data shown are means \pm SE of $n=4$ vesicle preparations. $* p<0.01$ vs. 0 and $* * p<0.001$ vs. 58 and 0 .

Thus according to (2) the estimated membrane potential difference at each of the different transmembrane $K^{+}$ gradients established in the different vesicles populations were: $0 \mathrm{mV}\left(1 \mathrm{mM} K^{+}\right.$inside and outside); $58 \mathrm{mV}\left(10 \mathrm{mM} K^{+}\right.$ outside and $1 \mathrm{mM} K^{+}$inside $)$and $116 \mathrm{mV}\left(100 \mathrm{mM} K^{+}\right.$outside and $1 \mathrm{mM} K^{+}$inside).

Fig. 5 shows the changes in fluorescence measured at the various $E_{m}$ in OMMV isolated from young rats. It shows that the more positive the $E_{m}$, the lower the change in 
fluorescence intensity upon vesicle addition. Furthermore there was a significant difference between the change in fluorescence intensity measured upon vesicle addition between each one of the different $K^{+}$gradients.

The effect of varying the concentration of gly-gln on the change in fluorescence $(\Delta F)$ measured when BBMV from young and middle-aged rats pre-equilibrated at $\mathrm{pH} 7.4$ were added to a cuvette containing DiS-C3-(3) and 100-1000 $\mu \mathrm{M}$ gly-gln at pH 6.0 is shown in Fig. 6. This shows that as the concentration of gly-gln increased so the $\Delta \mathrm{F}$ became progressively smaller suggesting the generation of an increasingly positive membrane potential difference consistent with a progressively larger magnitude of gly-gln uptake.

\section{DISCUSSION}

The renal proximal tubule is an important site of nutrient reabsorption, where many of the reabsorptive processes depend on the electrochemical gradient manifested by inward cation gradients and the membrane potential difference [3]. Therefore the development of methods that enable the accurate measurement of the membrane potential difference will provide important insights into the regulation of nutrient handling and waste disposal, and, because many pharmacological agents share similar transport mechanisms, yield greater knowledge regarding drug delivery and disposition.

In this study we have shown for the first time that the fluorescent indicator, 3'3'-dipropylthiacarbocyanine iodide (DiS- $\left.\mathrm{C}_{3}-(3)\right)$ can be used as an indicator of the membrane potential difference in membrane vesicles isolated from young and middle-aged rat kidneys. This was clear from

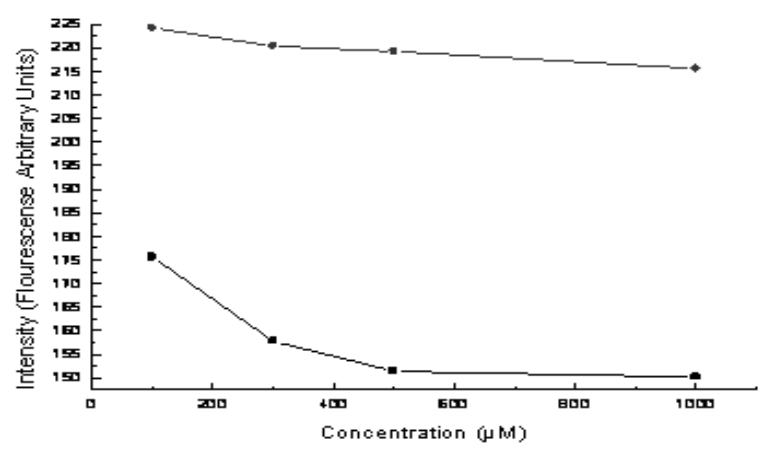

Fig. 6. Changes in fluorescence measured when BBMV isolated from young (lower line) and middle-aged (upper line) rats pre-equlibrated at $\mathrm{pH} 7.4$ were added to a cuvette containing $\mathrm{DiS}_{-} \mathrm{C}_{3}-(3)$ and $100-1000 \mu \mathrm{M}$ gly-gln at $\mathrm{pH}$ 6.0. Data shown are taken from a single experiment representative of four such experiments.

the changes in the fluorescence measured when vesicles pre-equilibrated with $1 \mathrm{mM}$ potassium were added to a cuvette containing different potassium concentrations where in each case the change in fluorescence depended on the membrane potential as predicted from the Nernst Equation (1) (Fig. 2-5). Furthermore the sensitivity of the reported changes in fluorescence at different membrane potentials were sufficient that DiS- $\mathrm{C}_{3}-(3)$ could also be applied to sense differences in the magnitude of gly-gln uptake for relatively small changes in substrate concentration (Fig. 6).

Sims et al demonstrated that when a population of artificial membrane vesicles was added to a mixture containing a cyanine dye such as DiS-C3-(5) that the dye distributed between the solution and the membranes according to the membrane potential difference [14]. They and others showed that as the membrane potential difference of the vesicles became more hyperpolarised more dye became associated with the membrane leading to the formation of dye aggregates which was measured as fluorescence quenching [10]-[11], [14]. In the thirty or so intervening years since that ground breaking report advances in fluorescence spectroscopy have improved the speed and resolution of fluorescence measurements so that it is now possible to measure dye incorporation into the membrane prior to quenching. This was apparent when vesicles pre-equilibrated with $1 \mathrm{mM}$ potassium were added to a cuvette containing $100 \mathrm{mM}$ potassium leading to the generation of a positive membrane potential of $116 \mathrm{mV}$ evoking less dye incorporation into the membrane and a smaller change in fluorescence upon vesicle addition compared to when the same vesicles were added to a cuvette containing either $1 \mathrm{mM}$ or $10 \mathrm{mM}$ potassium. Contrast for example the approximately 150,000 arbitrary units (AU) change in fluorescence $(\Delta F)$ in Fig. 2for a 1:1 gradient with the approximately 100,000 AU $\Delta \mathrm{F}$ for a 1:100 gradient in Fig. 4. Importantly when the mean $\Delta \mathrm{F}$ measured at each of the different potassium gradients were compared they were found to be significantly different. This suggests that the fluorescence of DiS-C3-(3) can be used to measure changes in membrane potential in membrane vesicles isolated from the kidneys of young and middle-aged rats.

Finally, in order to define the scope of potential applications of $\mathrm{DiS}_{-} \mathrm{C}_{3}-(3)$ it was considered important to show that it could discriminate between the relatively subtle changes in membrane potential that occur during cotransport activities. The transport of small peptides and peptidomimetic drugs by PEPT1 and PEPT2 in the renal proximal tubule is well known to be an electrogenic process where uptake of the substrate is accompanied by the translocation of hydrogen ions [4], [14]-[15]. Studies have also shown that dipeptide uptake is generally greatest when the extracellular $\mathrm{pH}$ is around 6.0 and the intracellular $\mathrm{pH}$ is in the normal range [16]-[19]. This is consistent with the hypothesis that the increasing magnitude of gly-gln uptake into BBMV as the extravesiculargly-gln increased was accompanied by a greater influx of protons leading to the generation of an increasingly positive membrane potential difference that was reflected in the smaller changes in fluorescence upon vesicle addition as shown in Fig. 6. According to the known distribution of the PEPT transporters in different segments of the proximal tubule the isoform most likely to be abundantly present in brush border membrane vesicles isolated from the superficial cortex is the lower affinity transporter, PEPT1 [3]. This may explain the seeming absence of any sign of saturation in the uptake of gly-gln in BBMV isolated from middle-aged rats (Fig. 6). Expanding the range of substrate concentrations may confirm whether saturation occurs for gly-gln transport in the BBMV isolated from young rats (Fig. 6). 


\section{CONCLUSION}

In this experiment, we have shown that the fluorescence of the dye, DiS- $\mathrm{C}_{3}-(3)$, can be used to measure changes in the membrane potential difference in membrane vesicles isolated from the kidneys of young and middle-aged rats. We have also shown howthe ability of $\mathrm{DiS}_{-} \mathrm{C}_{3}-(3)$ to sense the membrane potential difference can be applied to the measurement ofgly-gln transport. This is the first time that this particular dye has been used for this purpose. Since this method only requires detection equipment and the dye, DiS- $\mathrm{C}_{3}-(3)$, the potential use of this dye to measure changes in the membrane potential difference in membrane could be applied on a wide-range of biological and non-biological transports (e.g. nutrients, ions, and peptide-like drugs transports).

\section{ACKNOWLEDGMENT}

The authors would like to thank Dr Pierre Moens for advice andguidance involving fluorescence spectroscopy.

\section{REFERENCES}

[1] P.-Y. Chen, B. Joang, T. Shinohara, M. Das, Z. Chen, and S.-F. Lin, "The initiation of the heart beat," Circ. J. vol. 74, pp. 221-225. 2010.

[2] M. Hüttemann, I. Lee, A. Pecinova, P. Pecina, K. Przyklenk, and J. W. Doan, "Regulation of oxidative phosphorylation, the mitochondrial membrane potential, and their role in human disease," J Bioeng. Biomembr. vol. 40, pp. 445-456. 2008.

[3] L. D. Faller, "Mechanistic studies of sodium pump," Archiv Biochem. Biophys. vol. 476, pp. 12-21. 2008.

[4] M. Boll, M. Herget, M. Wagener, W. Weber, D. D. Markovich, J. Biber and W. Clauss, "Expression cloning and functional characterization of the kidney cortex high-affinity proton-coupled peptide transporter," Proc. Natl. Acad Sci. USA. vol. 93, pp. 284-289. 1996.

[5] H. Shen, D. E. Smith, T. Yang, Y. G. Huang, J. B. Schnermann, and F. C. Brosius, "Localization of PEPT1 and PEPT2 proton-coupled oligopeptide transporter mRNA and protein in rat kidney," Am. J. Physiol. vol. 276, pp. F658-F665. 1999.

[6] P. J. Schultheis, L. L. Clarke, P. Meneton, M. L. Miller, M. Soleimani, L. R. Gawenis, and T. M. Riddle, "Renal and intestinal absorptive defects in mice lacking the NHE3 Na+/H+ exchanger," Nat. Genet. vol. 19, pp. 282-285. 1998.

[7] C. M. H. Anderson and D. T. Thwaites, "Hijacking solute carriers for proton-coupled drug transport," Physiol. vol. 25, pp. 364-377. 2010.

[8] F. Viera and G. Malnic, "Hydrogen ion secretion by rat renal cortical tubules as studied by an antimony electrode," Am. J. Physiol. vol. 214, pp. 710-718. 1968

[9] C. D. A. Brown, N. King, and N. L. Simmons, "Co-expression of an anion conductance pathway with $\mathrm{Na}^{+}$-glucose cotransport in rat renal brush-border membrane vesicles," PflügersArchiv. vol. 423, pp. 406-410. 1993.

[10] G. Cassano, M. Maffia, V. Vilella, and C. Storelli, "Effects of membrane-potential on Na cotransports in eel intestinal brush-border membrane-vesicles - studies with a fluorescent dye," J. Membr. Biol. vol. 101, pp. 225-236. 1988.

[11] S. Vayro and N. L. Simmons, "An effect of $\mathrm{Ca}^{2+}$ on the intrinsic $\mathrm{Cl}^{-}$conductance of rat kidney cortex brush border membrane vesicles," J. Membr. Biol. vol. 150, pp. 163-173. 1996.

[12] M. Donnowitz, E. Emmer, J. McCullen, L. Reinlab, M. E. Cohen, R. P. Rood, J. Madara, G. W. G. Sharp, H. Murer, and K. Malmstrom, "Freeze-thaw and high voltage discharge allow macromolecule uptake into ileal brush-border vesicles," Am. J. Physiol. vol. 252, pp. G723-G735. 1987.

[13] P. J. Sims, A. S. Waggoner, C.-H. Wang, and J. F. Hoffman, "Studies of the mechanism by which cyanine dyes measure membrane potential in red blood cells and phosphatidylcholine vesicles," Biochem. vol. 13, pp. 3315-3329. 1974.

[14] H. Daniel and I. Rubio-Aliaga, "An update on renal peptide transporters,” Am. J. Physiol. vol. 284, pp. F885-F892. 2003.

[15] H. Daniel and G. Kottra, "The proton oligopeptidecotransporter family SLC15 in physiology and pharmacology," PflügersArchiv. vol. 447, pp. 610-618. 2004.

[16] H. Lin and N. King, "Demonstration of functional dipeptide transport with expression of PEPT2 in guinea pig cardiomyocytes," PflügersArchiv. vol. 453, pp. 915-922. 2007

[17] M. Brandsch, C. Brandsch, P. D. Prasad, V. Ganapathy, U. Hopfer, and F. H. Leibach, "Identification of a renal cell line that constitutively expresses the kidney-specific high-affinity H+/peptide cotransporter,' FASEB. vol. 9, pp. 1489-1496. 1995.

[18] Y.-J. Fei, Y. Kanai, S. Nussberger, V. Ganapathy, F. H. Leibach, M. F. Romero, S. K. Singh, W. F. Boron, and M. A. Hediger, "Expression cloning of a mammalian proton-coupled oligopeptide transporter," Nat. vol. 368, pp. 563-566. 1994.

[19] M. E. Ganapathy, M. Brandsch, P. D. Prasad, V. Ganapathy, and F. H. Leibach, "Differential recognition of $\beta$-lactam antibiotics by intestinal and renal peptide transporters, PEPT 1 and PEPT 2," J. Biol. Chem. vol. 270, pp. 25672-25677. 1995 